\title{
A EXPERIÊNCIA DOCENTE EM EAD ENQUANTO UM FATOR QUE CONTRIBUI PARA A ESCOLHA DAS TDICS
}

\author{
LONDRINA/PR MAIO/2018
}

\author{
Antônio Ricardo Catânio - UNOPAR - antonio.catanio@gmail.com \\ Eric Ferreira dos Santos - UNOPAR - eric_ferreira_net@hotmail.com \\ Luisa Maria Sarábia Cavenaghi - UNOPAR - luisasarabia@hotmail.com \\ Nádia Brunetta Peretti - UNOPAR - nabrunetta@yahoo.com.br \\ Emilia Yoko Okayama - UNOPAR - miliyoko@hotmail.com \\ Mônica Maria Silva - UNOPAR - mmsilva_1@yahoo.com.br \\ Luana Nascimento de Paula - UNOPAR - luananpaula@hotmail.com \\ Renata Storti Pereira - UNOPAR - restortip@gmail.com \\ Eduardo Camargo de Aguiar - UNOPAR - eduardocaguiar2@gmail.com
}

Tipo: Relato de Experiência Inovadora (EI)

Categoria: Métodos e Tecnologias

Setor Educacional: EDUCAÇÃO SUPERIOR

RESUMO

Este artigo tem como proposta relatar as experiências docentes, considerando a importância das Tecnologias Digitais e de Comunicação (TDICs) no ambiente acadêmico, e mais especificamente, durante a condução de um projeto de pesquisa proposto por docentes de uma Instituição de Ensino Superior (IES) privada, sediada no norte do Paraná, com a participação de alunos voluntários matriculados em cursos de graduação dos polos de apoio presencial distribuídos em território nacional. Dentre os achados da pesquisa, o AVA e o aplicativo WhatsApp se destacaram, sendo utilizados para aperfeiçoar a interação e o vínculo entre os alunos e os professores. Concluiu-se que há a intenção e a disposição dos professores em utilizarem outras TDICs, como as redes sociais, ao longo do projeto, visando estreitar o relacionamento com os alunos pesquisadores em atividades de iniciação científica desenvolvidas na modalidade EaD.

Palavras-chave: TDIC. Tecnologia Digital de Informação e Comunicação. EaD. Relato de experiência. Projeto de Pesquisa. 


\section{Introdução}

A Educação à Distância (EaD) evoluiu substancialmente a partir da segunda metade do século XX (ERBS, 2004). No Brasil, os últimos anos tem apontado para um crescimento acelerado desta modalidade, o número de matrículas em cursos de graduação à distância era de aproximadamente 1,5 milhões em 2016 , isto é, cerca de $18,6 \%$ dos oito milhões de universitários EaD no país (SATHLER, 2017). A modalidade surgiu como uma alternativa para melhorar a qualidade de vida das pessoas, especialmente no que tange ao acesso no ensino superior, pois permitiu expressivas possibilidades à moradores de regiões afastadas e com difícil acesso as universidades (SOUZA, 2016).

No âmbito da educação, são relevantes as discussões acerca do uso de tecnologias de informação e comunicação (ABDULRAHMAN; TTADROS, ALLAWZI, 2012). Assim, a $\mathrm{EaD}$ adquiriu características relacionadas a busca pela qualidade, agilidade, expansão e inclusão global, uma vez que se aliou às Tecnologias Digitais de Informação e Comunicação (TDICs) (SOUZA, 2016; ABDULRAHMAN et al., 2012).

Por ocupar uma posição de destaque na sociedade, a educação busca acompanhar as mudanças trazidas pela globalização sobre as tecnologias de informação e comunicação, isto é, visa acompanhar o ritmo dos avanços das TDICs (YAMAGUCHI; MILL, 2016; ASSARE, MOHAMMADI, FOROUTAN et al., 2015).

A revolução da informação também impulsiona as instituições de ensino para a adoção das TDICs (ABDULRAHMAN et al., 2012). Contudo, embora isto figure pela admissão das tecnologias, o ambiente de educação contribui para a escolha de recursos tecnológicos adequados (CASTILHO, 2014), uma vez que os reflexos da globalização têm uma relação próxima com a educação (ASSARE et al., 2015).

Diante do exposto, este trabalho tem por objetivo relatar as experiências docentes, considerando a importância das TDICs no ambiente acadêmico, e mais especificamente, durante a condução de um projeto de pesquisa proposto por docentes de uma Instituição de Ensino Superior (IES) privada, sediada no norte do Paraná, com a participação de alunos voluntários matriculados em cursos de graduação em polos de apoio presencial distribuídos em território nacional. O projeto de pesquisa em questão retrata um estudo sobre consultoria em gestão empresarial de micro e pequenas empresas brasileiras.

Tendo em vista a necessidade de exposição das experiências docentes, realizou-se o presente relato de experiência. $O$ relato de experiência é um recurso de pesquisa 
descritiva, o qual expressa uma reflexão sobre uma ação ou um conjunto de ações que abordam uma situação vivenciada na esfera profissional de interesse para a comunidade cientifica (CAVALCANTE; LIMA, 2012).

\section{Tecnologias Digitais de Informação e Comunicação (TDICs)}

A gestão das informações pelas pessoas demanda a aplicação de tecnologias, as quais, para ser efetiva, requer Tecnologias Digitais de Informação e Comunicação (TDICs) que transmitam as informações com tempestividade, atendendo as necessidades informacionais de seus usuários (OLUWAFEMI, 2015).

Para Afonso (2002), a difusão da informática e da internet nas décadas de 1970 e 1980 levou os especialistas a adotarem duas expressões: TIC e TDICs. A sigla TIC refere-se a Tecnologia da Informação e Comunicação e representa "as tecnologias digitais de informática e de redes de troca de dados" (AFONSO, 2002, p. 169). Contudo, para o autor, a sigla mais adequada seria TDIC, por reportar-se aos aspectos tecnológicos de informação e comunicação, pois, segundo ele, as tecnologias de informação e comunicação existem desde tempos remotos (AFONSO, 2002).

A Internet é uma das ferramentas que possibilita a qualquer indivíduo ser produtor de um conteúdo em diversos formatos (vídeo, áudio, relatos, artigos, entre outros) permitindo amplo acesso a quem interessar, alterando a forma como as pessoas, empresas e instituições se relacionam e comunicam-se (GARCIA, 2016).

Dessa forma, as TDICs promovem a captura, a transmissão e a exibição de dados e informações por meio eletrônico e, especialmente na área da educação, fornecem uma base sólida, útil para a capacitação, desenvolvimento e ampliação das competências, pois envolvem os meios de comunicação, as redes computacionais, as tecnologias multimídia, as bibliotecas eletrônicas, o ensino à distância, as salas virtuais, etc. (DEBANDE; OTTERSTEN, 2004).

Alguns benefícios são esperados pelo uso das TDICs, tais como: auxiliar o aluno na execução de atividades; ordenar a aprendizagem, levando à definições de problemas, questionamentos, métodos de organização, avaliações e a geração de conhecimento; permitir aos professores a comunicação por meio de textos informativos na web, gráficos, sons e vídeos; possibilitar o acesso e investigação rápida de fatos e ideias, resolvendo problemas e chegando a conclusões; permitir o acesso e a utilização de conteúdos em várias aplicações em diferentes localidades (DEBANDE; OTTERSTEN, 2004). 
Neste sentido, um dos recursos comumente utilizados em um contexto de ensino à distância é o Ambiente Virtual de Aprendizagem (AVA). O AVA é considerado um elemento notável de toda a estrutura tecnológica que é utilizada para suportar a realização da $\mathrm{EaD}$, uma vez que permite a disponibilização dos conteúdos digitais dos cursos e as interações entre os participantes (COELHO; TEDESCO, 2017).

Sousa, Moita e Carvalho (2011) entendem que a educação, para ser de qualidade, precisa estar voltada para a aprendizagem. No caso da $\mathrm{EaD}$, isto fica centrado na modelagem do AVA, na mediação pedagógica e no planejamento das atividades que valorizem as ações interativas, viabilizados pelos recursos tecnológicos (SOUSA et al. 2011). Dessa forma, as TDICs conduzem às mudanças, porque, conforme sugere Gaible e Burns (2005), a criação de planejamentos para ações no AVA e o desenvolvimento ou aprimoramento de atividades possibilitam testá-los em campo para verificar seus impactos.

O uso das TDICs também se aplica à projetos de pesquisas por facilitar a conexão entre diferentes pessoas e possibilitam a expansão do escopo da pesquisa sem restrição geográfica (LATCHEM, 2010). Isto posto, segundo Pinheiro (2003), a utilização das TDICs pode ser considerada tanto na fase da pesquisa propriamente dita, como também no compartilhamento de informações e do conhecimento produzido ao final da pesquisa. Assim, as TDICs podem contribuir para que os programas de educação possuam maior eficácia, alcançando cada vez maior número de comunidades e regiões (TAKAHASHI, 2000).

Nesta acepção, e-mails, intranet, chat, recursos online e mídias sociais ou Social Media Apps (SMA) são utilizados no âmbito profissional, seja pelo computador, tablet ou smartphone para auxiliar na comunicação (SANTOS; RODRIGUES; MELO, 2017). Cabe ressaltar que a facilidade das redes sociais, tais como o Instagram, o Twitter, o Facebook, ou de aplicativos de comunicação como o WhatsApp, contribui para a utilização de diferentes linguagens e a difusão de ideias e conteúdo, potencializando o uso das TDICs na educação em várias regiões (LUCENA, 2016).

Percebe-se que as ações relacionadas ao uso das TDICs apresentam desafios durante o gerenciamento do AVA. A realização de treinamentos por meio das TDICs oportuniza a implementação de cronogramas e metodologias, a orientação dos alunos, o desenvolvimento de materiais e o estimulo à aprendizagem individual e coletiva (LATCHEM, 2010). Para o aluno, o treinamento é essencial, pois contribui para o manuseio dos recursos, a obtenção de conhecimentos e a prática sólida por relacionar as tecnologias e a interação humana com a aprendizagem prática, o desenvolvimento 
de competências e as confianças no uso das TDICs no AVA (STEKETEE, 2005; LATCHEM, 2010).

\section{Relato de experiência}

$\mathrm{Na}$ sociedade atual, os recursos tecnológicos favorecem ao processo de ensino e aprendizagem. De acordo com Castilho (2014), na educação o próprio ambiente acadêmico contribui para a escolha desses recursos. Dentro desse contexto, serão apresentadas no decorrer desse estudo as evidências sobre a importância do uso de TDICs adequadas, para o desenvolvimento de um projeto de pesquisa em uma instituição de ensino superior privada.

Os docentes do projeto iniciaram as atividades divulgando o edital de pesquisa e as instruções para inscrição de alunos voluntários. Foi utilizado como recursos de divulgação o: a) flyer eletrônico com as informações do projeto; b) edital de abertura no AVA; e c) e-mail informativo para divulgar o flyer, as instruções de acesso, o cadastro no projeto. Entretanto, durante o acompanhamento das inscrições, identificou-se um número reduzido de interessados. Nesse sentido, foi elaborado: d) o vídeo de divulgação, armazenado no Youtube, cujo link foi disponibilizado via e-mail ao públicoalvo. Com isso, percebeu-se um aumento na adesão do projeto.

Após o encerramento da divulgação do projeto, iniciou-se o processo de validação dos inscritos por meio do uso de planilhas eletrônicas. As funções das planilhas foram de serventia para identificar dados ou informações necessárias não cadastradas durante o processo. Findo a validação, os alunos passaram a receber interações para os primeiros contatos via e-mail, prosseguindo para as capacitações.

No ambiente virtual do projeto foram disponibilizados os materiais de capacitação para as etapas seguintes da pesquisa. Tais capacitações incluíam vídeos evidenciando os seguintes assuntos: a) iniciação científica; b) elaboração do currículo lattes; e c) coleta de dados. Durante os vídeos, a equipe de professores instruiu os alunos quanto ao uso da plataforma lattes para a criação do currículo do aluno e quanto ao uso dos Formulários Google para a coleta de dados.

Os alunos apresentaram várias dificuldades para acessar o AVA e durante a realização das atividades. Tais contratempos foram atendidos por meio de e-mail e via Chats semanais, os quais visavam também estreitar o relacionamento com os alunos. Neste sentido, o uso do chat facilitava o esclarecimento de dúvidas e auxiliava nas contribuições entre os participantes, aperfeiçoando a interação e celeridade entre 
professores e alunos. Além disso, no decorrer do atendimento aos alunos, os professores ficaram responsáveis por grupos de alunos, reduzindo-se, assim, o tempo de resposta das dúvidas que surgissem.

Ao final dos treinamentos, os alunos voluntários tiveram que executar o estágio da coleta de dados. Assim, foi disponibilizado aos participantes um formulário eletrônico para ser aplicado nas empresas. Após o trabalho em campo, os alunos comunicaram os dados obtidos das empresas participantes aos professores orientadores via e-mail. Em seguida, os docentes validaram os dados coletados por meio das planilhas eletrônicas, uma vez que os dados coletados eram nelas armazenados. Em caso de identificação de dado ausente, os professores entraram em contato com os alunos por meio de ligações telefônicas e e-mails.

Ainda nessa etapa, ficou evidente a dificuldade de alguns alunos. Nesse caso, os professores buscaram por outras TDICs além do uso de chats e e-mail. Tais recursos tecnológicos incluem o aplicativo WhatsApp, o qual permitiu retornos imediatos, inclusive de alguns discentes que não tinham respondido nenhum e-mail. Também, recorreu-se ao uso de contato telefônico, o qual possibilitou um retorno positivo com alunos, especialmente em relação aos que moravam em regiões afastadas, tendo apenas acesso à internet no dia de aulas no polo de apoio presencial.

Dentre as tecnologias utilizadas no projeto, destacou-se o uso frequente do WhatsApp, uma vez que o mesmo permitiu o retorno tempestivo das informações acerca do projeto e dos alunos, a criação de vínculos por meio de diálogos entre os envolvidos e uma relação de confiança entre orientador-aluno. Assim, para a continuidade do projeto com o uso das TDICs, os professores perceberam que a transmissão da informação ocorre em menor tempo e, para as próximas fases da pesquisa, consideram utilizar novas TDICs, tais como as plataformas de redes sociais.

\section{A relevância da tecnologia na EaD}

Diante do relato apresentado, é possível observar diversos aspectos relacionados às TDICs e os seus efeitos na interação entre os envolvidos. Os professores demonstram a importância do uso das TDICs no desenvolvimento da pesquisa em várias etapas do projeto. Isto fica evidente ao analisar o uso das ferramentas de comunicação, como chats e fóruns, que possibilitaram o compartilhamento de informações.

Pinheiro (2003) confirma a importância das TDICs para contribuir na comunicação entre os pesquisadores, pois ajudam na superação das distâncias geográficas, tida como um 
fator considerável no uso dessas tecnologias em países como o Brasil.

Durante a execução do projeto de pesquisa, além do envio de e-mails e ligações telefônicas, o AVA foi utilizado para melhorar a interação entre os participantes, sendo isso um elemento evidente, como retrata Coelho e Tedesco (2017). Isso ocorreu de forma efetiva, sendo que foram disponibilizados instruções e materiais para capacitar os alunos, incluindo vídeos, salas de chat e fórum de interação. Esse resultado positivo em relação a interatividade proporcionada pelo AVA se comprova na literatura, pois Cavalcanti (2014) afirma que o AVA é um meio de interatividade que faz com que as pessoas se interessem cada vez mais em estudar com a modalidade EaD, onde o aluno pode encontrar as informações necessárias.

Souza (2016) corrobora a respeito da importância do AVA, já que, no cenário da pesquisa, o ambiente é um relevante aliado para apresentação dos conteúdos de forma aberta e irrestrita, isto é, é uma ferramenta que está a? disposição da educação para gerar um padrão de pesquisa e ensino de qualidade, com o uso das TDICs.

Pelo relato do projeto, observa-se que a utilização de recursos de interação individualizados como a comunicação mediada por telefone ou por meio do aplicativo WhatsApp gera resultados positivos. Deste modo, a comunicação mediada por Social Media Apps (SANTOS et al., 2017), tal como o WhatsApp, produz efeitos melhores, pois permite a comunicação e a interatividade de forma direta.

O WhatsApp foi a ferramenta que mais se destacou no projeto, visto que o retorno era muito rápido e os alunos criaram um vínculo maior com seus orientadores. Esse tipo de experiência se confirma na literatura já que, de acordo com Santos, Rodrigues e Mello (2017, p.2) o "Whatsapp é um exemplo de aplicativo que tem se destacado quanto à adesão de usuários tanto para uso pessoal quanto para uso profissional". De acordo com os autores, o uso do aplicativo alcançou a marca de 100 milhões, no ano de 2015 (SANTOS et al., 2017).

À vista disso, Lucena (2016) observa que os estudantes estão imersos nas tecnologias digitais, utilizando com facilidade as redes sociais como Instagram, Facebook e WhatsApp para produzirem linguagens e difundirem as suas ideias, o que potencializa o uso das TDICs na educação.

\section{Conclusões e sugestões para pesquisas futuras}

Este trabalho teve por objetivo relatar as experiências docentes, considerando a 
importância das TDICs no ambiente acadêmico, e mais especificamente, durante a condução de um projeto de pesquisa proposto por docentes de uma Instituição de Ensino Superior (IES) privada, sediada no norte do Paraná, com a participação de alunos voluntários matriculados em cursos de graduação dos polos de apoio presencial distribuídos em território nacional.

Por meio das informações relatadas, constatou-se que há a intenção e a disposição dos professores em utilizarem outras TDICs, como as redes sociais, ao longo do projeto, visando aumentar o vínculo entre alunos e professores e agilizar o retorno sobre as atividades desenvolvidas.

Os achados neste estudo revelam as fragilidades encontradas durante as atividades de pesquisa, realizadas no ambiente da $\mathrm{EaD}$, e a importância do uso de diversos recursos tecnológicos aplicáveis à diferentes realidades, considerando inclusive o perfil dos alunos, para o pleno desenvolvimento das habilidades requeridas a um pesquisador. Destaca-se também a importância da interação e mediação constante para o estímulo ao desenvolvimento de autonomia dos alunos, principalmente no que concerne à aplicação da pesquisa em campo e a coleta de dados.

Assim, diante das discussões, surgem novas oportunidades para outras pesquisas, tais como: os fatores que contribuam para a escolha das TDICs no ensino superior e as maneiras que ampliam o seu uso durante as orientações acadêmicas.

\section{REFERÊNCIAS}

ABDULRAHMAN, Eman J.; TTADROS, Ibrahem H.; ALLAWZI, Sulieman. The role of communication and information technology in the development of university management at governmental jordanian universities from the academic leader's point of view. Information and Knowledge Management, v. 2, n. 8, 2012.

AFONSO, Carlos A. Internet no Brasil: alguns dos desafios a enfrentar. Informática Pública, v. 4, n. 2, p. 169-184, 2002.

ASSARE, A. R.; MOHAMMADI, M.; FOROUTAN, M.; SALEHIZADEH, M. The Impact of Globalization on Education. Journal of Administrative Management, Education and Training, v. 12, p. 27-33, 2015.

CARVALHO, A. B. Os múltiplos papéis do professor em educação a distância: uma abordagem centrada na aprendizagem. In: Anais... 18 encontro de pesquisa 
educacional do norte e nordeste - EPENN. Maceió, 2007.

CASTILHO, Luciane Barbosa. O uso da tecnologia da informação da informação e comunicação (TIC) no processo de ensino e aprendizagem no ensino superior brasileiro. Dissertação (Mestrado Profissional) - Curso em Sistemas de Informação e Gestão do Conhecimento), FUMEC, Belo Horizonte, 2014.

CAVALCANTE, Bruna Luana de Lima; LIMA, Uirassú Tupinambá Silva de. Relato de experiência de uma estudante de Enfermagem em um consultório especializado em tratamento de feridas. Journal of Nursing and Health, Pelotas, v. 1, n. 2, p. 94-103, jan/jul 2012.

CAVALCANTI, Carolina Magalhães Costa. Design Thinking como metodologia de pesquisa para concepção de um Ambiente Virtual de Aprendizagem centrado no usuário. SIED: EnPED-Simpósio Internacional de Educação a Distância e Encontro de Pesquisadores em Educação a Distância, 2014.

COELHO, Willyans Garcia; TEDESCO, Patricia Cabral de Azevedo Restelli. A percepção do outro no ambiente virtual de aprendizagem: presença social e suas implicações para Educação a Distância. Revista Brasileira de Educação, v. 22, n. 70, 2017.

DEBANDE, Olivier; OTTERSTEN, Eugenia Kazamaki. Information and Communication Technologies. Higher Education Management and Policy, v. 16, n. 2, p. 31-61, 2004.

ERBS, Rita Tatiana Cardoso. 0 processo de avaliação na modalidade de educação a distância. Rio Grande do Sul: Universidade de Caxias do Sul, 2004.

GAIBLE, Edmond; BURNS, Mary. Using Technology to Train Teachers: Appropriate Uses of ICT for Teacher Professional Development in Developing Countries. Online Submission, 2005.

GARCIA, J. L. Sistemas de Informação de Marketing. São Paulo: Pearson Education do Brasil, 2016.

LATCHEM, Colin. Using ICT to train teachers in ICT. Open and Distance Learning, $\mathrm{p}$. 75, 2010.

LUCENA, Simone. Culturas digitais e tecnologias móveis na educação. Educar em 
Revista, Curitiba, Brasil, n. 59, p. 277-290, jan./mar. 2016.

OLUWAFEMI, Olatunji. The impact of information communication technology on small and medium scale enterprise productivity in Nigeria. Business Economics and Tourism. 2015.

PINHEIRO, Lena Vania Ribeiro. Comunidades científicas e infra-estrutura tecnológica no Brasil para uso de recursos eletrônicos de comunicação e informação na pesquisa. Ciência da Informação, v. 32, n. 3, p. 62-73, dez. 2003.

SANTOS, Ana Cristina Batista dos; RODRIGUES, Márcia Carolina Araújo; MELO, Wendel Carlos Carvalho. O Trabalho Mediado por Tecnologias da Informação e Comunicação: Uso Corporativo do Aplicativo Whatsapp. In: Encontro de Administração da Informação, 4, 2017, Curitina. Anais... Curitiba: ANPAD, 2017.

SATHLER, Luciano. Educação Aberta e Flexível: um caminho sem volta. ABED Associação Brasileira e Educação a Distância, 2018. Disponível em: <http://www.abed.or g.br/site/pt/midiateca/textos_ead/1469/2017/12/educacao_aberta_e_flexivel\%2c_um_ca minho_sem_volta>. Acesso em 07 de mai. 2018.

STEKETEE, Carole. Integrating ICT as an integral teaching and learning tool into preservice teacher training courses. Issues in Educational Research, v. 15, n. 1, p. 101, 2005.

SOUSA, Robson Pequeno de. MOITA, Filomena M. C. da S. C. CARVALHO, Beatriz Gomes Carvalho. (Orgs.) Tecnologias digitais na educação. Campina Grande: EDUEPB, 2011.

SOUZA, R. A. Multimídia no EaD. Sa?o Paulo: Cengage, 2016.

TAKAHASHI, T. (Org). Sociedade da informação no Brasil: livro verde. Brasília: Ministério da Ciência e Tecnologia, 2000.

YAMAGUCHI, Rubens; MILL, Daniel. Estudo Sobre Sistemas de Educação a Distância: Materiais Didáticos e das Tecnologias de suporte em foco. In: Simpósio Internacional de Educação A Distância e Encontro de Pesquisadores em Educação a Distância, São Carlos. Anais... São Carlos, 2016. 